gr-qc/0301106

\title{
SCALAR FIELD IN A MINIMALLY COUPLED BRANE WORLD: NO-HAIR AND OTHER NO-GO THEOREMS
}

\author{
K.A. Bronnikov ${ }^{a, b, 1}$, S.B. Fadeev ${ }^{a}$ and A.V. Michtchenko ${ }^{c, 2}$ \\ a VNIIMS, 3-1 M. Ulyanovoy St., Moscow 117313, Russia \\ ${ }^{b}$ Institute of Gravitation and Cosmology, PFUR, 6 Miklukho-Maklaya St., Moscow 117198, Russia \\ c SEPI-ESIME, IPN, Zacatenco, México, D.F., CP07738, Mexico
}

\begin{abstract}
In the brane-world framework, we consider static, spherically symmetric configurations of a scalar field with the Lagrangian $(\partial \phi)^{2} / 2-V(\phi)$, confined on the brane. We use the $4 \mathrm{D}$ Einstein equations on the brane obtained by Shiromizu et al., containing the conventional stress tensor $T_{\mu}^{\nu}$, the tensor $\Pi_{\mu}^{\nu}$ which is quadratic in $T_{\mu}^{\nu}$, and $E_{\mu}^{\nu}$ describing interaction with the bulk. For models under study, the tensor $\Pi_{\mu}^{\nu}$ has zero divergence, allowing one to consider $E_{\mu}^{\nu}=0$. Such a brane, whose $4 \mathrm{D}$ gravity is decoupled from the bulk geometry, may be called minimally coupled. Assuming $E_{\mu}^{\nu}=0$, we try to extend to brane worlds some theorems valid for scalar fields in general relativity (GR). Thus, the list of possible global causal structures in all models under consideration is shown to be the same as is known for vacuum with a cosmological constant in GR: Minkowski, Schwarzschild, (anti-)de Sitter and Schwarzschild-(anti-)de Sitter. A no-hair theorem, saying that, given a potential $V \geq 0$, asymptotically flat black holes cannot have nontrivial external scalar fields, is proved under certain restrictions. Some objects, forbidden in GR, are allowed on the brane, e.g, traversable wormholes supported by a scalar field, but only at the expense of enormous matter densities in the strong field region.
\end{abstract}

\section{Introduction}

The widely discussed brane-world models suppose that the observable Universe is a kind of domain wall, or brane, in a multidimensional space, with large or even infinite extra dimensions. The standard-model fields are supposed to be confined on the brane while gravity propagates in the surrounding bulk. The history of such models is traced back at least to the early 80s [1, cetrain progress then followed (see, e.g., 2]), but the recent outburst of interest owes to the progress in string theory and M-theory, in particular, to the Horava-Witten 11-dimensional model [3]. The brane-world approach has suggested a natural mechanism of solving the hierarchy problem of particle physics [4, 5, 6], preserving a correct behaviour of weak gravity on the brane. Many related important problems of cosmology and particle physics have also been addressed, see the recent reviews [7.

Most of modern studies consider a simplified version of this picture, the so-called RS2 class of models $[6$ gravity acts in a 5-dimensional bulk while our 4-dimensional world is a time-evolving 3-brane which supports all standardmodel fields. Gravity on the brane is described by modified 4-dimensional Einstein equations [8] [Eqs. (11) in Sec. 2] which contain additional terms leading to many new predictions as compared to general relativity (GR). One such term, the traceless tensor $E_{\mu}^{\nu}$, originating from the $5 \mathrm{D}$ Weyl tensor and describing tidal effects on the brane from the bulk geometry, may contribute to "dark energy" in cosmology [7] and leads to numerous new black hole [9] 10] and wormhole [11] solutions on the brane. Another additional term, $\Pi_{\mu}^{\nu}$, which is quadratic in the stress-energy tensor (SET) of matter confined on the brane, contributes to the cosmological evolution [7] and can lead to new strong field effects in local gravitational fields. Like other models, brane-world cosmologies often involve scalar fields with various potentials [7, 12, 13, which strongly affect the cosmological dynamics and are probably necessary for describing inflation and dark energy. Less is known about the properties of scalar fields in the physics of local objects on the brane.

In this paper, we consider the modified Einstein equations for static, spherically symmetric configurations of scalar fields with arbitrary potentials $V(\phi)$ in the absence of other kinds of matter (scalar-vacuum configurations, for short) and try to extend to brane worlds some theorems valid for scalar fields in GR. For minimally coupled scalar fields described by the Lagrangian $L_{s}=\frac{1}{2}(\partial \phi)^{2}-V(\phi)$, among such theorems are:

(i) The no-hair theorems 14, 15]: in case $V \geq 0$, as well as for some partly negative potentials, an asymptotically flat black hole cannot have a nontrivial external scalar field.

\footnotetext{
${ }^{1}$ e-mail: kb@rgs.mccme.ru

${ }^{2}$ e-mail: mial@maya.esimez.ipn.mx
} 
(ii) The "generalized Rosen theorem" saying that particlelike scalar-vacuum solutions (asymptotically flat solutions with a regular centre) are absent as long as $V(\phi) \geq 0$ (see [16] and references therein).

(iii) The nonexistence theorem for regular solutions without a centre (e.g., wormholes) [17.

(iv) The causal structure theorem [17, asserting that the list of possible types of global causal structures (and the corresponding Carter-Penrose diagrams) for scalar field configurations with any potentials $V(\varphi)$ and any spatial asymptotics is the same as is known for vacuum with a cosmological constant in GR.

Extensions of these theorems to scalar-tensor and Kaluza-Klein type multidimensional theories have been considered before 18, 19, 20, 16. For scalar vacuum in a brane world, the situation is complicated by the non-closed nature of the $4 \mathrm{D}$ equations (11): the "tidal" tensor $E_{\mu}^{\nu}$ bears information on the bulk gravity; moreover, in the general case there is stress-energy exchange between $E_{\mu}^{\nu}$ and matter on the brane represented by the quadratic tensor $\Pi_{\mu}^{\nu}$. Fortunately, for a scalar field in the static, spherically symmetric case $\Pi_{\mu}^{\nu}$ is conservative, making it possible to put $E_{\mu}^{\nu} \equiv 0$. This assumption closes the set of $4 \mathrm{D}$ field equations. Such a brane may be called minimally coupled: 4-dimensional gravity on the brane is then decoupled from the bulk geometry. For $T_{\mu}^{\nu}=0$, the $4 \mathrm{D}$ gravity equations reproduce the vacuum Einstein equation, and, for $T_{\mu}^{\nu} \neq 0$, the only possible brane-world effect is connected with the tensor $\Pi_{\mu}^{\nu}$, quadratic in $T_{\mu}^{\nu}$ and significant in strong fields.

A full description of the model evidently requires knowledge of the whole 5D geometry. Any specific solution on the brane must be "evolved" into the bulk, which is a nontrivial problem even for very simple brane metrics [9, 21, 22. We will not discuss the possible bulk properties of models in question and only note that the existence of the corresponding solutions to the higher-dimensional equations of gravity (in our case, the 5D vacuum Einstein equations with a cosmological term) is guaranteed by the Campbell-Magaard type embedding theorems 23. A recent discussion of these theorems, applied, in particular, to brane world scenarios, and more references can be found in Ref. [24].

In what follows, considering scalar fields on a minimally coupled brane, we shall see that the above theorems do not always hold even in this idealized case. The causal structure theorem proves to be valid without change. Bekenstein's 14. no-hair theorem for scalar fields with potentials $V=V\left(\phi^{2}\right)$ such that $d V / d\left(\phi^{2}\right) \geq 0$ also remains valid. On the contrary, solitonic (particlelike) solutions for $V \geq 0$ as well as wormholes cannot be excluded. Wormhole throats are admissible in a strong field region, and this circumstance does not allow us to prove the no-hair theorem for scalar fields with arbitrary nonnegative potentials in its full generality: one cannot exclude a black hole with scalar hair whose event horizon is located beyond a throat. However, an estimate based on an experimental restriction on the bulk length scale shows that both wormhole throats and particlelike solutions require energy densities and pressures far beyond the nuclear density.

\section{Field equations and conservation laws}

The gravitational field on the brane is described by the modified Einstein equations derived by Shiromizu, Maeda and Sasaki [8] from 5-dimensional gravity with the aid of the Gauss and Codazzi equations ${ }^{3}$ :

$$
G_{\mu}^{\nu}=-\Lambda_{4} \delta_{\mu}^{\nu}-\kappa_{4}^{2} T_{\mu}^{\nu}-\kappa_{5}^{4} \Pi_{\mu}^{\nu}-E_{\mu}^{\nu},
$$

where $G_{\mu}^{\nu}=R_{\mu}^{\nu}-\frac{1}{2} \delta_{\mu}^{\nu} R$ is the $4 \mathrm{D}$ Einstein tensor, $\Lambda_{4}$ is the $4 \mathrm{D}$ cosmological constant expressed in terms of the $5 \mathrm{D}$ cosmological constant $\Lambda_{5}$ and the brane tension $\lambda$ :

$$
\Lambda_{4}=\frac{1}{2}\left(\Lambda_{5}+\frac{1}{6} \kappa_{5}^{4} \lambda^{2}\right) ; \quad \kappa_{4}^{2}=8 \pi G_{\mathrm{N}}=\kappa_{5}^{4} \lambda /(6 \pi),
$$

where $\kappa_{4}$ is the $4 \mathrm{D}$ gravitational constant, $G_{\mathrm{N}}$ is Newton's constant of gravity; $T_{\mu}^{\nu}$ is the SET of matter confined on the brane; $\Pi_{\mu}^{\nu}$ is a tensor quadratic in $T_{\mu}^{\nu}$, obtained from matching the $5 \mathrm{D}$ metric across the brane:

$$
\Pi_{\mu}^{\nu}=\frac{1}{4} T_{\mu}^{\alpha} T_{\alpha}^{\nu}-\frac{1}{2} T T_{\mu}^{\nu}-\frac{1}{8} \delta_{\mu}^{\nu}\left(T_{\alpha \beta} T^{\alpha \beta}-\frac{1}{3} T^{2}\right)
$$

where $T=T_{\alpha}^{\alpha}$; lastly, $E_{\mu}^{\nu}$ is the "electric" part of the 5D Weyl tensor projected onto the brane: in proper 5D coordinates, $E_{\mu \nu}=\delta_{\mu}^{A} \delta_{\nu}^{C(5)} C_{A B C D} n^{B} n^{D}$ where $A, B, \ldots$ are $5 \mathrm{D}$ indices and $n^{A}$ is the unit normal to the brane. By construction, $E_{\mu}^{\nu}$ is traceless, $E_{\mu}^{\mu}=0$ [8].

\footnotetext{
${ }^{3}$ The sign conventions are: the metric signature $(+---)$; the curvature tensor $R_{\mu \rho \nu}^{\sigma}=\partial_{\nu} \Gamma_{\mu \rho}^{\sigma}-\ldots$, so that, e.g., the Ricci scalar $R>0$ for de Sitter space-time, and the stress-energy tensor (SET) such that $T_{t}^{t}$ is the energy density.
} 
The contracted Bianchi identities $\nabla_{\nu} G_{\mu}^{\nu}=0$ lead to the corresponding relation for the right-hand side of (1). Assuming the conservation law $\nabla_{\nu} T_{\mu}^{\nu}=0$ for matter, we obtain the condition

$$
\nabla_{\nu}\left(\Pi_{\mu}^{\nu}+E_{\mu}^{\nu}\right)=0
$$

but $\Pi_{\mu}^{\nu}$ and $E_{\mu}^{\nu}$, taken separately, are not necessarily divergence-free: there is, in general, stress-energy exchange between the brane matter and the bulk gravity.

Consider now a minimally coupled scalar field confined on the brane, with the 4D Lagrangian

$$
L_{s}=\frac{1}{2}(\partial \phi)^{2}-V(\phi),
$$

where $(\partial \phi)^{2}=g^{\mu \nu} \phi_{, \mu} \phi_{, \nu}$. The field equation and the tensor $T_{\mu}^{\nu}$ have the conventional form

$\nabla^{\nu} \nabla_{\nu} \phi+d V / d \phi=0$

$T_{\mu}^{\nu}=\phi_{, \mu} \phi^{, \nu}-\frac{1}{2} \delta_{\mu}^{\nu}(\partial \phi)^{2}+\delta_{\mu}^{\nu} V$.

The tensor $T_{\mu}^{\nu}$ is conservative while the corresponding $\Pi_{\mu}^{\nu}$ is, in general, not:

$$
\begin{aligned}
\Pi_{\mu}^{\nu} & =\phi_{, \mu} \phi^{, \nu}\left[\frac{5}{12}(\partial \phi)^{2}-\frac{7}{6} V\right]+\delta_{\mu}^{\nu}\left[-\frac{1}{16}(\partial \phi)^{4}-\frac{5}{12} V(\partial \phi)^{2}+\frac{17}{12} V^{2}\right] ; \\
\nabla_{\nu} \Pi_{\mu}^{\nu} & =\frac{1}{3} \phi^{, \nu} \phi^{, \alpha}\left(\phi_{, \mu} \phi_{; \alpha \mu}-\phi_{, \nu} \phi_{; \alpha \mu}\right)+\frac{1}{4} V \phi^{, \alpha} \phi_{; \alpha \mu}+\frac{1}{2} V \phi_{, \mu} \nabla^{\nu} \nabla_{\nu} \phi .
\end{aligned}
$$

For static, spherically symmetric configurations, the brane metric can be written in the form

$$
d s^{2}=A(\rho) d t^{2}-\frac{d \rho^{2}}{A(\rho)}-r^{2}(\rho) d \Omega^{2}
$$

where $d \Omega^{2}=d \theta^{2}+\sin ^{2} \theta d \varphi^{2}$ is the linear element on a unit sphere and $\rho$ is the radial coordinate under the convenient "quasiglobal" gauge $g_{00} g_{11}=-1$. With this metric, the scalar field $\phi(\rho)$ has the SET

$$
T_{\mu}^{\nu}=\frac{1}{2} \operatorname{diag}(f,-f, f, f)+\delta_{\mu}^{\nu} V(\phi), \quad f \stackrel{\text { def }}{=} A(\rho) \phi^{\prime 2},
$$

where the prime denotes $d / d \rho$. The corresponding $\Pi_{\mu}^{\nu}$ has the nonzero components

$$
\Pi_{0}^{0}=\Pi_{2}^{2}=\Pi_{3}^{3}=\frac{1}{48}\left(4 V^{2}+4 f V-3 f^{2}\right) ; \quad \Pi_{1}^{1}=\frac{1}{48}\left(4 V^{2}-4 f V+f^{2}\right),
$$

and a direct inspection leads to the important conclusion that the tensor $\Pi_{\mu}^{\nu}$ is conservative in the static, spherically symmetric case: $\nabla_{\nu} \Pi_{\mu}^{\nu}=0$.

This means, in particular, that the assumption $E_{\mu}^{\nu}=0$ does not contradict the field equations for scalar fields on the brane. Under this assumption, gravity on the brane decouples from the bulk gravity, and Eqs. (19), (6) comprise a closed set of equations. They can be written as follows:

$$
\begin{aligned}
\left(A r^{2} \phi^{\prime}\right)^{\prime} & =r^{2} d V / d \phi ; \\
\frac{1}{2 r^{2}}\left(A^{\prime} r^{2}\right)^{\prime} & =-\Lambda_{4}-\kappa_{4}^{2} V+\frac{\kappa_{5}^{4}}{48}\left(4 V^{2}-A^{2} \phi^{\prime 4}\right) ; \\
2 \frac{r^{\prime \prime}}{r} & =-\kappa_{4}^{2} \phi^{2}\left[1+\frac{\kappa_{5}^{4}}{12 \kappa_{4}^{2}}\left(2 V-A \phi^{\prime 2}\right)\right] ; \\
A\left(r^{2}\right)^{\prime \prime}-r^{2} A^{\prime \prime} & =2 . \\
\frac{1}{r^{2}}\left(-1+A^{\prime} r r^{\prime}+A r^{\prime 2}\right) & =-\Lambda_{4}+\frac{1}{2} \kappa_{4}^{2}\left(A \phi^{\prime 2}-2 V\right)-\frac{\kappa_{5}^{4}}{48}\left(A \phi^{\prime 2}-2 V\right)^{2} .
\end{aligned}
$$

Only three of the five equations are independent, in particular, (17) is a first integral of (14)- (16). Given a potential $V(\varphi)$, this is a determined set of equations for the unknowns $r, A, \varphi$.

The scalar-vacuum equations of GR are restored in case $\kappa_{5}=0$.

\section{No-go theorems}

Let us try to extend some theorems known in GR to a minimally coupled brane world, using Eqs. (13)-(17). 


\subsection{Causal structure theorem}

The first theorem concerns the possible number and order of Killing horizons, coinciding with the number and order of zeros of $A(\rho)$ at finite $r$. A simple (first-order) or any odd-order horizon separates a static region, $A>0$ (also called an $\mathrm{R}$ region), from a nonstatic region, $A<0$, where (10) is a homogeneous cosmological metric of Kantowski-Sachs type (a T region). A horizon of even order separates regions with the same sign of $A(\rho)$. The gauge $g_{00} g_{11}=-1$ makes it possible to jointly consider regions on both sides of a horizon since, as is directly verified, $\rho$ behaves in its neighbourhood like a manifestly regular Kruskal-like coordinate.

The disposition of horizons unambiguously determines the global causal structure of space-time, up to identification of isometric surfaces, if any. The following theorem severely restricts such possible dispositions.

Theorem 1. Consider solutions to Eqs. (13)-(17). Let there be a static region $a<\rho<b \leq \infty$. Then:

(i) all horizons are simple;

(ii) no horizons exist at $\rho<a$ and at $\rho>b$.

A proof of this theorem is the same as in Ref. 17 and rests on the property $T_{0}^{0}=T_{2}^{2}$ of the SET (11), leading to the same property of the tensor $\Pi_{\mu}^{\nu}$. The idea is that Eq. (16), expressing the equality $R_{0}^{0}=R_{2}^{2}$, can be rewritten as

$$
r^{4} B^{\prime \prime}+4 r^{3} r^{\prime} B^{\prime}=-2
$$

where $B(\rho)=A / r^{2}$. At points where $B^{\prime}=0$, we have $B^{\prime \prime}<0$, therefore $B(\rho)$ cannot have a regular minimum. So, having once become negative while moving to the left or to the right along the $\rho$ axis, $B(\rho)$ (and hence $A(\rho)$ ) cannot return to zero or positive values.

By Theorem 1, there can be at most two simple horizons around a static region. A second-order horizon separating two nonstatic regions can appear, but this horizon is then unique, and the model has no static region.

The possible dispositions of zeros of the function $A(\rho)$, and hence the list of possible global causal structures, are thus the same as in the case of spherically symmetric vacuum in GR with a cosmological constant. The types of Carter-Penrose diagrams that are possible with solutions to Eqs. (13)-(16) are exhausted by the diagrams known for different particular cases of the Schwarzschild-de Sitter solution, described by the metric (10) with $r \equiv \rho$ and $A=1-2 m / r-\Lambda_{4} r^{2} / 3$. The only structure types are Minkowski, Schwarzschild, de Sitter, AdS, and Schwarzschild(A)dS, see Refs. [25] 18, 19, 20] for more detail. Asymptotically flat black holes, if any, have the Schwarzschild structure.

Theorem 1 is independent of any assumptions on the shape of the potential and on the spatial asymptotics.

\subsection{Theorems which fail in a brane world}

The absence of wormhole throats (i.e., regular minima of $r$ ) for scalar-vacuum configurations in GR follows from Eq. (15) which, in case $\kappa_{5}=0$, leads to $r^{\prime \prime} \leq 0$, so that a regular minimum of $r(\rho)$ is impossible. However, in case $\kappa_{5} \neq 0$ the r.h.s. of Eq. (15) can become positive in a strong-field region, therefore wormhole throats and wormholes as global scalar-vacuum solutions on the brane are not excluded.

Consider now Eqs. (13) - (17) in case $\Lambda_{4}=0$, which is a natural assumption when dealing with local objects, very small on the cosmological scale. It is then natural to study asymptotically flat configurations. A flat asymptotic $\rho \rightarrow \infty$ is characterized by

$$
r \approx \rho, \quad A=1-2 G_{\mathrm{N}} m / \rho+o\left(\rho^{-1}\right), \quad \phi=o\left(\rho^{-3 / 2}\right), \quad V(\phi)=o\left(\rho^{-3}\right),
$$

where $m$ is the Schwarzschild mass. According to Eq. (13), at a flat asymptotic $d V / d \phi=o\left(\rho^{-5 / 2}\right)$, so that both $V$ and $d V / d \phi$ vanish at infinity. These are minimal requirements that follow directly from the field equations. If, in addition, we assume that $V(\phi)$ is at least $C^{2}$-smooth and $\phi$ can be expanded in powers of $1 / \rho$, it follows that $d V / d \phi$ must vanish at large $\rho$ as $\rho^{-4}$ or more rapidly.

The nonexistence theorem for particlelike solutions (i.e., asymptotically flat solutions with a regular centre) follows for scalar vacuum in GR from a universal identity [16, valid for any static, spherically symmetric particlelike solution and obtained by comparing two expressions for the mass $m$. One of them is the standard integral $m=4 \pi G \int T_{0}^{0} r^{2} d r$, the other follows from Tolman's well-known formula, and their comparison leads to the identity

$$
\int_{\rho_{c}}^{\infty}\left[\left(r^{\prime}-1\right) T_{0}^{0}+T_{1}^{1}+T_{2}^{2}+T_{3}^{3}\right] r^{2} d \rho=0
$$

where $\rho_{c}$ is the value of $\rho$ at the centre. Applied to a scalar field with the SET (11), Eq. (20) takes the form

$$
\int_{\rho_{c}}^{\infty}\left[f+2 V\left(2+r^{\prime}\right)\right] r^{2} d \rho=0
$$


In GR we always have $r^{\prime}(\rho) \geq 1$ due to $r^{\prime}(\infty)=1$ and $r^{\prime \prime} \leq 0$, hence (21) cannot be satisfied without assuming $V<0$ at least in some range of $\rho$.

For a particlelike solution on the brane the identity (20) is also valid but with $T_{\mu}^{\nu}$ replaced by $T_{\mu}^{\nu}+\Pi_{\mu}^{\nu}$, and instead of 21] we obtain

$$
\int_{\rho_{c}}^{\infty}\left\{\kappa_{4}^{2}\left[f+2 V\left(2+r^{\prime}\right)\right]+\frac{1}{24} \kappa_{5}^{4}\left[(f-2 V)^{2} r^{\prime}+8 V^{2}-2 f^{2}\right]\right\} r^{2} d \rho=0
$$

As we saw, $r(\rho)$ now can have a minimum, so we cannot rule out $r^{\prime}<0$ and even $r^{\prime}<-2$ in a certain region of space; moreover, the integrand in (22) contains the term $-2 f^{2}$. Therefore (22) can hold with nonnegative $V$.

\subsection{No-hair theorems}

Let us continue studying asymptotically flat solutions and try to extend the no-scalar-hair theorem to brane worlds.

First of all we notice that Eq. (13) is the same as in GR, therefore Bekenstein's argument 14 that rests solely on the scalar field equation in a black hole geometry and does not employ the equations of gravity, is applicable here without change and can only be slightly refined in our spherically symmetric case due to Theorem 1 . Let us reproduce it in our notation.

Theorem 2. Given a potential $V=V\left(\phi^{2}\right)$ such that $d V / d\left(\phi^{2}\right) \geq 0$, the only asymptotically flat black hole solution to Eqs. (13)-(16) is characterized by $V \equiv 0, \phi=$ const and the Schwarzschild metric in the whole domain of outer communication.

Proof. Let $\rho=h$ be the event horizon, so that $A(h)=0$ and $r(h)$ is finite, and let $\rho \rightarrow \infty$ be a flat asymptotic, so that $A(\rho)>0$ in the range $h<\rho<\infty$. Multiplying Eq. (13) by $\phi$, we can rewrite it in the form

$$
\left(r^{2} A \phi^{\prime 2}\right)^{\prime}-r^{2} A \phi^{\prime 2}=r^{2} \phi \frac{d V}{d \phi},
$$

which gives after integration from $h$ to infinity:

$$
\int_{h}^{\infty}\left(A \phi^{2}+\phi \frac{d V}{d \phi}\right) r^{2} d \rho=\left.r^{2} A \phi \phi^{\prime}\right|_{h} ^{\infty}
$$

At the flat asymptotic, according to (19), $\phi \phi^{\prime}=o\left(r^{-2}\right)$, so that $\left.r^{2} A \phi \phi^{\prime}\right|^{\rho \rightarrow \infty}=0$. Furthermore, as $\rho \rightarrow h$, $A \sim \rho-h$ since, by Theorem 1 , it is a simple horizon. A horizon is a regular surface, at which $f=A \phi^{\prime 2}$ must be finite (otherwise the components of $T_{\mu}^{\nu}$ would blow up, leading, due to gravity equations, to a singular geometry). Therefore, even if $\phi^{\prime}$ blows up as $\rho \rightarrow h+0$, it cannot grow faster than $(\rho-h)^{-1 / 2}$, so that $|\phi(h)|<\infty$. As a result, we have $A \phi \phi^{\prime}=0$ at $\rho=h$, the whole r.h.s. of Eq. (24) is zero, making the integral in the l.h.s. vanish.

By assumption, $\phi V_{\phi}=2 \phi^{2} d V / d\left(\phi^{2}\right) \geq 0$, so the integrand in (24) is nonnegative and, to produce a zero integral, it must vanish identically for $h<\rho<\infty$ : we must have $\phi=$ const and (since $V \rightarrow 0$ at the asymptotic) $V \equiv 0$.

Note that Theorem 2 did not need the condition $V \geq 0$, but only $d V / d\left(\phi^{2}\right) \geq 0$. The theorem even holds for some partly negative potentials. A good example is $V=K\left(\phi^{2}-\eta^{2}\right)^{3}$ with positive constants $K$ and $\eta$. Indeed, both $V$ and $d V / d \phi$ vanish at $\phi= \pm \eta$, making $V(\phi)$ compatible with a flat asymptotic; $d V / d\left(\phi^{2}\right)=3 K\left(\phi^{2}-\eta^{2}\right)^{2} \geq 0$, the condition of Theorem 2 is satisfied; and $V<0$ at $-\eta<\phi<\eta$.

In Ref. 14] Theorem 2 was proved in a more general setting, for static asymptotically flat black holes without the spherical symmetry assumption.

Now, assuming a general nonnegative potential, let us prove the following theorem, using an argument somewhat similar to that of Adler and Pearson [15].

Theorem 3. Given a potential $V(\phi) \geq 0$, the only asymptotically flat black hole solution to Eqs. (13)-(16), such that $r^{\prime}>0$ at and outside the event horizon, is characterized by $\phi=$ const and the Schwarzschild metric in the whole domain of outer communication.

Proof. Let again $\rho=h$ be the black hole event horizon and $h<\rho<\infty$ the domain of outer communication. Consider the function

$$
\mathcal{F}_{1}(\rho)=\frac{r^{2}}{r^{\prime}}\left[2 V-A \phi^{\prime 2}\right]
$$


which is everywhere meaningful due to our assumption $r^{\prime}>0$. Let us find $\mathcal{F}_{1}^{\prime}$, substituting $\phi^{\prime \prime}$ from (13), $r^{\prime \prime}$ from (15) and $A^{\prime}$ from (17). The result is

$$
\mathcal{F}_{1}^{\prime}(\rho)=\mathcal{F}_{2}(\rho) \stackrel{\text { def }}{=} r\left[4 V+\frac{\phi^{\prime 2}}{r^{\prime 2}}+A \phi^{\prime 2}+\frac{\kappa_{5}^{2}}{48} \frac{r^{2} \phi^{\prime 2}}{r^{\prime 2}}\left(2 V-A \phi^{\prime 2}\right)^{2}\right],
$$

or, after integration from $h$ to infinity,

$$
\mathcal{F}_{1}(\infty)-\mathcal{F}_{1}(h)=\int_{h}^{\infty} \mathcal{F}_{2}(\rho) d \rho
$$

Evidently, $\mathcal{F}_{1}(\infty)=0$ due to the asymptotic flatness conditions (19).

Consider $\mathcal{F}_{1}(h)$. The metric regularity at the horizon requires $r^{\prime}(h)<\infty$, as is easily verified by calculating the Kretschmann scalar $R^{\alpha \beta \gamma \delta} R_{\alpha \beta \gamma \delta}$ (see, e.g., [16), therefore the quantity $\mathcal{F}_{1}(h)$ is, in general, finite but nonzero. If, however, we admit a nonzero value of $A \phi^{\prime 2}$ at $\rho=h$ whereas $A \sim \rho-h$, so that $\phi^{\prime} \sim(\rho-h)^{1 / 2}$, then the integral in (27) will diverge at $\rho=h$ due to the second term in brackets in (26), and this will in turn lead to an infinite value of $\mathcal{F}_{1}(h)$. We have to conclude that $A \phi^{\prime 2} \rightarrow 0$ as $\rho \rightarrow h$, and consequently

$$
\mathcal{F}_{1}(h)=\frac{2 r^{2}(h)}{r^{\prime}(h)} V(h) \geq 0 .
$$

Thus the l.h.s. of Eq. (27) is nonpositive and the r.h.s. is nonnegative. The only way to satisfy (27) is to put $V \equiv 0$ and $\phi^{\prime} \equiv 0$ in the whole range $\rho>h$, and the only solution for the metric is then Schwarzschild.

The above reasoning does not work if we admit $r^{\prime} \leq 0$ at some $\rho \geq h$. Suppose that $r^{\prime}=0$ at some $\rho_{1}>h$ and $r^{\prime}<0$ at $h \leq \rho<\rho_{1}$. Then, even if $\phi^{\prime}$ turns to zero together with $r^{\prime}$, so that Eq. (27) remains meaningful, the l.h.s. in (27), equal to $-V(h) / r^{\prime}(h)$, is positive in case $V(h)>0$, and Eq. (27) may hold. However, a horizon located at a wormhole throat, such that $r^{\prime}>0$ at $\rho>h$ and $r^{\prime}(h)=0$, is impossible. Indeed, we then obtain $-\mathcal{F}_{1}(h+0)=-\infty$ for $V(h)>0$ and a nonpositive limiting value $-\mathcal{F}_{1}(h+0)$ in case $V(h)=0$.

\subsection{Numerical estimates}

Using an observational restriction on the bulk energy scale $m_{5}$ and the well-known value of the $4 \mathrm{D}$ Planck energy, $m_{4}=\kappa_{4}^{-1}=\left(8 \pi G_{\mathrm{N}}\right)^{-1 / 2} \approx 3.7 \cdot 10^{18} \mathrm{GeV}$, we can estimate the density scale of brane matter (scalar field in our case) which can lead to a geometry drastically different from GR predictions.

Namely, consider the conditions on a wormhole throat: $r>0, r^{\prime}=0$ and $r^{\prime \prime} \geq 0$. It is this phenomenon that restricts the validity of Theorem 3, to say nothing on the possible existence of wormholes. As follows from Eq. (15), the quantity $A \phi^{2}-2 V>0$ at the throat (we suppose $\phi^{\prime} \neq 0$ ). Then, the l.h.s. of Eq. (17) is simply $-r^{-2}$ at the throat, and the condition that the r.h.s. is negative yields (assuming, as before, $\Lambda_{4}=0$ )

$$
W:=\left.\left(A \phi^{\prime 2}-2 V\right)\right|_{\text {throat }} \gtrsim W_{-}=\frac{24 \kappa_{4}^{2}}{\kappa_{5}^{4}}=24 m_{4}^{4}\left(\frac{m_{5}}{m_{4}}\right)^{6}
$$

where $m_{4}^{4} \approx 0.75 \cdot 10^{91} \mathrm{~g} \mathrm{~cm}^{-3}$ is the Planck density. Note that $W / 2=-T_{1}^{1}$ is the radial pressure of the scalar field. The energy density is $T_{0}^{0}=\frac{1}{2} A \phi^{2}+V$, and $T_{0}^{0}>W / 2$ if $V>0$. Then $W / 2$ can be used as a lower bound for $T_{0}^{0}$. If we even admit $V<0$ such that $T_{0}^{0} \ll W$ near the throat, the estimate of $W$ will still refer to radial pressure.

A similar estimate can be obtained for a possible particlelike solution in case $V \geq 0$. Indeed, Eq. (22) can only hold if the integrand is negative in some region. If there is no wormhole throat (i.e., the above estimate does not work) and $r^{\prime}>0$ in the whole space, this may happen at the expense of the last term $-2 f^{2}$ which means that, at least, $f \equiv A \phi^{\prime 2}>12 \kappa_{4}^{2} / \kappa_{5}^{4}=\frac{1}{2} W_{-}$. A particlelike solution with $V \geq 0$ thus requires a sufficiently high kinetic energy density $\frac{1}{2} A \phi^{\prime 2}>\frac{1}{4} W_{-}$in a certain region of space; due to $V \geq 0$, the full density $\frac{1}{2} f+V$ will be still larger.

The quantity (28) is extremely sensitive to the value of $m_{5}$. Thus, it has been claimed on the basis of accelerator data that the bulk energy scale $m_{5}$ might be as small as a few TeV. Taking, for certainty, $m_{5}=10 \mathrm{TeV}$, we obtain $m_{5} / m_{4} \approx 2.7 \cdot 10^{-15}$, and consequently $W_{-} \approx 7.3 \cdot 10^{4} \mathrm{~g} \mathrm{~cm}^{-3}$. This is smaller than the mean density of white dwarfs $\left(5 \div 6 \cdot 10^{5} \mathrm{~g} \mathrm{~cm}^{-3}[26\right.$.

A much tighter restriction follows from the recent short-range Newtonian gravity tests [27]: the length scale $\ell=\left(6 /\left|\Lambda_{5}\right|\right)^{1 / 2} \lesssim 1 \mathrm{~mm}$. Eqs. (2) with $\Lambda_{4}=0$ then lead to

$$
\frac{m_{5}}{m_{4}}=\left(\pi \ell m_{4}\right)^{-1 / 3}=\left(\frac{l_{\text {Planck }}}{\ell}\right)^{1 / 3}
$$


whence $\left(m_{5} / m_{4}\right)^{3} \gtrsim 2.6 \cdot 10^{-32}$, and $W \gtrsim 10^{29} \mathrm{~g} \mathrm{~cm}^{-3}$, an enormous value, many orders of magnitude over the nuclear density.

We can conclude that as long as the scalar field density and pressure are much smaller than this value of $W$, wormhole throats cannot appear, and all the above no-go theorems, known for a scalar field in GR, remain valid in a minimally coupled brane world.

\section{Concluding remarks}

We have seen that some no-go theorems for scalar vacuum hold in GR but can be violated in a minimally coupled brane world. In particular, the no-hair theorem for asymptotically flat black holes holds only under the additional assumption that there is no wormhole throat in the domain of outer communication. Accordingly, static scalar vacuum in a brane world can in principle yield as many as three kinds of solutions impossible in GR: (i) traversable wormholes, (ii) particlelike solutions for scalar fields with $V \geq 0$ and (iii) black holes with scalar hair, whose horizons are located beyond wormhole throats. All of them become possible due to the strong field behaviour of brane-world gravity, more precisely, due to the properties of the tensor $\Pi_{\mu}^{\nu}$. An estimate shows, however, that such exotic objects can only form by a scalar field whose energy density (and/or radial pressure) are many orders of magnitude larger than the density of an atomic nucleus $\left(\sim 10^{15} \mathrm{~g} \mathrm{~cm}^{-3}\right)$.

The above results can be readily generalized in two respects: for brane worlds of dimensions greater than four and for scalar field multiplets $\left\{\phi^{a}\right\}$ with Lagrangians of sigma-model type

$$
L_{\sigma}=h_{a b}(\phi) g^{\mu \nu} \partial_{\mu} \phi^{a} \partial_{\nu} \phi^{b}-V(\phi),
$$

where $h_{a b}(\phi)$ is a positive-definite matrix of functions of $\phi^{a}$.

If we abandon the minimal coupling assumption $E_{\mu}^{\nu}=0$, then a possible nonzero $E_{\mu}^{\nu}$ brings about much ambiguity. Thus, in isotropic cosmology it adds "dark radiation" of arbitrary density (one extra constant) [7, 12. In static spherical symmetry it adds an arbitrary function of the radial coordinate [11, 28, 10]; in particular, vacuum black holes and wormholes may be obtained with any functions $g_{00}(r)$ satisfying the proper boundary conditions [11, 10]. This is the case in the simplest RS2-type brane world models Ref. [6], possessing a single extra dimension, $\mathbb{Z}_{2}$ symmetry with respect to the brane and no matter in the bulk, to say nothing of more complex models. The latter may include scalar fields in the bulk [29, multiple (at least two) branes [30, timelike extra dimensions, lacking $\mathbb{Z}_{2}$ symmetry, a 4D curvature term [13, etc; further references may be found in the cited papers and the reviews [7]. To improve the predictive power of brane world scenarios, it seems necessary to remove the "redundant freedom", applying reasonable physical requirements such as regularity and stability to complete multidimensional models.

The recent paper by Anderson and Tavakol [31] (which appeared when this study was completed) shows that, even in the RS2 framework, different characterizations of the bulk lead to different forms of the effective 4D equations, though "all characterizations are equivalent if one considers the entire brane-bulk system". In our view, the 4D gravity equations on the brane, which determine the observable picture of the Universe, must also be unique in a given bulk-brane system, and their formulation (11) seems to be preferable due to a clear distinction of the bulk Weyl tensor contribution $E_{\mu}^{\nu}$ and the tensor $\Pi_{\mu}^{\nu}$ quadratic in $T_{\mu}^{\nu}$, important in strong fields. Other formulations [31] probably mix these contributions. The problem evidently needs a further study.

\section{Acknowledgements}

KB and SF acknowledge partial financial support from the RFBR Grant 01-0217312a, the Russian Ministry of Education and the Russian Ministry of Industry, Science and Technologies.

\section{References}

[1] V.A. Rubakov and M.E. Shaposhnikov, Phys. Lett. 152B, 136 (1983); I. Antoniadis, Phys. Lett. 246B, 377 (1985);

M. Visser, Phys. Lett. 159B, 22 (1985);

M. Pavšič, Phys. Lett. 116A, 1 (1986) gr-qc/0101075; Nuovo Cim. A 95, 297 (1986).

[2] M. Akama, Prog. Theor. Phys. 78, 184 (1987);

G.W. Gibbons and D.L. Wiltshire, Nucl. Phys. B 287, 717 1987;

M. Pavšič, Grav. EG Cosmol. 2, 1 (1996); 3, 305 (1997).

[3] P. Horava and E. Witten, Nucl. Phys. B 460, 506 (1996); ibid., 475, 94 (1996).

[4] N. Arkani-Hamed, S. Dimopoulos and G.R. Dvali, Phys. Lett. 429B, 263 (1998);

I. Antoniadis, N. Arkani-Hamed, S. Dimopoulos and G.R. Dvali, Phys. Lett. 436B, 257 (1998), hep-ph/9804398 
[5] L. Randall and R. Sundrum, Phys. Rev. Lett. 83, 3370 (1999), hep-ph/9905221

[6] L. Randall and R. Sundrum, Phys. Rev. Lett. 83, 4690 (1999), hep-ph/9906064

[7] V.A. Rubakov, "Large and infinite extra dimensions", Phys. Usp. 44, 871 (2001); hep-ph/0104152

R. Maartens, "Geometry and dynamics of the brane world", gr-qc/0101059

D. Langlois, "Gravitation and cosmology in a brane universe", gr-qc/0207047

S. Nojiri, S.D. Odintsov and S. Ogushi, Int. J. Mod. Phys. A 17, 4809 (2002), hep-th/0205187

Ph. Brax and C. van de Bruck, "Cosmology and brane worlds: a review", hep-th/0303095

[8] T. Shiromizu, K. Maeda and M. Sasaki, Phys. Rev. D 62, 024012 (2000).

[9] A. Chamblin, S.W. Hawking and H.S. Reall, Phys. Rev. D 61, 065007 (2000);

A. Chamblin, H.S. Reall, H. Shinkai and T. Shiromizu, Phys. Rev. D 63, 064015 (2001), hep-th/0008177

R. Casadio, A. Fabbri and L. Mazzacurati, Phys. Rev. D 65, 084040 (2001), gr-qc/0111072

N. Dadhich, S. Kar, S. Mukherjee and M. Visser, Phys. Rev. D 65, 064004 (2002).

[10] K.A. Bronnikov, H. Dehnen and V.N. Melnikov, "On a general class of black holes in a brane world", gr-qc/0304068

[11] K.A. Bronnikov and S.-W. Kim, "Possible wormholes in a brane world", gr-qc/0212112

[12] J.E. Lidsey, T. Matos and L.A. Ureña-López, "The inflaton field as self-interacting dark matter", astro-ph/0111292

A.V. Toporensky, P.V. Tretyakov and V.O. Ustiansky, "New properties of scalar field dynamics in brane isotropic cosmological models", gr-qc/0207091

[13] Yu.V. Shtanov, hep-ph/0108153

V. Sahni and Yu.V. Shtanov, "New vistas in braneworld cosmology", gr-qc/0205111

[14] J.D. Bekenstein, Phys. Rev. D 5, 1239 (1972); ibid., 2403; "Black holes: classical properties, thermodynamics, and heuristic quantization", in: "Cosmology and Gravitation", ed. M. Novello, Atlantisciences, France, 2000, pp. 1-85; gr-qc/9808028 (review).

[15] S. Adler and R.B. Pearson, Phys. Rev. D 18, 2798 (1978).

[16] K.A. Bronnikov, S.B. Fadeev and A.V. Michtchenko, "Scalar field in multidimensional gravity. No-hair and other no-go theorems", Gen. Rel. Grav. 35, 505 (2003), gr-qc/0212065

[17] K.A. Bronnikov, Phys. Rev. D 64, 064013 (2001).

[18] K.A. Bronnikov and G.N. Shikin, Grav. \& Cosmol 8, 107 (2002), gr-qc/0109027

[19] K.A. Bronnikov, Acta Phys. Polon. B32, 3571 (2001), gr-qc/0110125

[20] K.A. Bronnikov, J. Math. Phys. 43, 6096 (2002), gr-qc/0204001

[21] R. Casadio and L. Mazzacurati, "Bulk shape of brane-world black holes", gr-qc/0205129

[22] T. Wiseman, Phys. Rev. D 65, 124007 (2002).

[23] J. Campbell, "A Course of Differential Geometry", Clarendon, Oxford, 1926; L. Magaard, PhD thesis, Kiel, 1963.

[24] S.S. Seahra and P.S. Wesson, "Application of the Campbell-Magaard theorem to higher-dimensional physics", gr-qc/0302015

[25] K. Lake and R. Roeder, Phys. Rev. D 15, 3513 (1977);

M. Katanaev, T. Klösch and W. Kummer, Ann. Phys. (USA) 276, 191 (1999).

[26] C.W. Allen, "Astrophysical Quantities", Athlone Press, London, 1973.

[27] J.S. Long et al., Nature 421, 922 (2003);

J.S. Long and J.C. Price, "Current short-range tests of the gravitational inverse square law", hep-ph/0303057

[28] M. Visser and D.L. Wiltshire, "On-brane data for brane-world stars", hep-th/0212333

[29] K. Maeda and D. Wands, Phys. Rev. D 62, 124009 (2000), hep-th/0008188

T. Shiromizu, T. Torii and D. Ida, JHEP 0203 (2002) 007, hep-th/0105256

[30] S. Kanno and J. Soda, Phys. Rev. D 66, 043526 (2002), hep-th/0207029

T. Shiromizu and K. Koyama, "Low-energy effective theory for two-brane system — covariant curvature formulation", hep-th/0210066

[31] E. Anderson and R. Tavakol, "Ambiguities in formulation of GR-based brameworld models", gr-qc/0305013 